\title{
Effects of a quark-model-based nucleon- $\Delta$ potential on the two-nucleon system above pion threshold
}

\author{
A. Valcarce and F. Fernández \\ Grupo de Física Nuclear, Universidad de Salamanca, E-37008 Salamanca, Spain \\ H. Garcilazo, M. T. Peña, ${ }^{*}$ and P. U. Sauer \\ Institute for Theoretical Physics, University Hannover, D-3000 Hannover 1, Germany
}

(Received 8 February 1993; revised manuscript received 15 December 1993)

\begin{abstract}
The interaction between a nucleon and a $\Delta$ isobar is studied. The nucleon- $\Delta$ potential is derived from a nonrelativistic quark model. It is compared with a corresponding one based on meson exchange. The quark-model potential is strongly repulsive at small relative distances. The effects of the potential on elastic and inelastic nucleon-nucleon and pion-deuteron scattering are calculated. Some observables show sensitivity with respect to the chosen nucleon- $\Delta$ potential.
\end{abstract}

PACS number(s): 13.75.Cs, 21.30.+y, 21.45.+v, 21.60.Gx

\section{INTRODUCTION}

The $\Delta$ isobar is an important mode of nucleonic excitation. It is seen as a resonance in pion-nucleon scattering and photopion and electropion production from the nucleon. In nuclear structure, the $\Delta$ isobar is an agent for corrections $[1,2]$ in the traditional picture of the nucleus as a system of nucleons only: It is considered a nuclear constituent in addition to nucleons. Its excitation in the nucleus yields many-nucleon forces and many-nucleon currents for electroweak processes. In nuclear reactions, the $\Delta$ isobar provides a mechanism $[3,4]$ for pion scattering, pion production, and pion absorption.

The interaction of the $\Delta$ isobar with nucleons is experimentally unobservable and theoretically unknown to a large extent. The conceptual consistency between the $\Delta$ isobar, bound in nuclear systems, and the pion-nucleon $P_{33}$ resonance, embedded in the nuclear medium, can only be given by models for the structure of the isobar and resonance. Thus the $\Delta$ isobar's effects on nuclear observables have to be studied theoretically and are model dependent. This paper explores the properties of the two-nucleon system above the pion threshold in their dependence on a chosen nucleon- $\Delta$ potential.

The paper uses two models for the nucleon- $\Delta$ potential. One model is based on nonrelativistic quark dynamics between composite baryons, the other on meson exchange between elementary baryons. The predictions for observables of the two-nucleon system above the pion threshold arising from the two different potential models are compared. Section II describes the employed potentials in configuration space and discusses their similarities and differences. Section III recalls the technical apparatus for calculating the observables of elastic and inelastic

\footnotetext{
*Present address: Centro de Física Nuclear da Universidade de Lisoba, P-1699 Lisboa Codex, Portugal, and CEBAF, 12000 Jefferson Ave., Newport News, VA 23606.
}

nucleon-nucleon and pion-deuteron scattering. Section IV presents the results and conclusions.

\section{POTENTIAL BETWEEN A NUCLEON AND A $\triangle$ ISOBAR}

The nucleon- $\Delta$ potential is not directly accessible in experiments. Nevertheless, there are attempts to extract nucleon- $\Delta$ phase shifts and other features of the nucleon$\Delta$ interaction from data $[5,6]$. However, the extracted properties of the nucleon- $\Delta$ interaction are rather model dependent without rigorous theoretical meaning. This section compares two theoretical models for a nucleon- $\Delta$ potential. In both models, the $\Delta$ isobar is considered a stable baryon. In the potential based on meson exchange, the $\Delta$ isobar is elementary as the nucleon $(N)$ is, differing from the nucleon by its mass, quantum numbers, meson coupling constants, and form factors. In the potential based on nonrelativistic quark dynamics, the nucleon and $\Delta$ isobar are both composite. However, the decay of the $\Delta$ isobar to pion-nucleon states is not incorporated in the considered quark model; thus, the $\Delta$ isobar is also a stable baryon in the considered quark description.

\section{A. Potential based on nonrelativistic quark dynamics}

The nucleon- $\Delta$ potential is derived from nonrelativistic quark dynamics. The employed quark model works with quark degrees of freedom only, the gluon ones being frozen into effective quark masses $m_{q}$ and into effective quark potentials $\mathcal{V}_{q q}$ of two-body nature. The quark model is that of Ref. [7]. Up and down quarks are considered only. The quark masses are assumed to be isospin independent.

The quark Hamiltonian $\mathcal{H}_{q}$ is $\mathcal{H}_{q}=\mathcal{H}_{q 0}+\mathcal{V}_{q q}$, with $\mathcal{H}_{q 0}$ denoting the kinetic energy. The quark-quark po- 
tential $\mathcal{V}_{q q}$ has four distinct parts, i.e.,

$$
\mathcal{V}_{q q}=\mathcal{V}_{\mathrm{OGE}}+\mathcal{V}_{\text {con }}+\mathcal{V}_{\mathrm{OSE}}+\mathcal{V}_{\mathrm{OPE}}
$$

$\mathcal{V}_{\text {OGE }}$ being the Fermi-Breit one-gluon exchange, $\mathcal{V}_{\text {con }}$ being a quadratic confinement potential, and $\mathcal{V}_{\text {OSE }}$ and
$\mathcal{V}_{\text {OPE }}$ being the one-meson exchange of a sigma $(\sigma)$ and of a pion $(\pi)$ between quarks, respectively. The four parts of the potential are used in nonrelativistic approximation; they become local in that approximation and are employed in the forms

$$
\begin{gathered}
\mathcal{V}_{\mathrm{OGE}}\left(\mathbf{r}_{i j}\right)=\frac{1}{4} \alpha_{s} \boldsymbol{\lambda}(i) \cdot \boldsymbol{\lambda}(j)\left\{\frac{1}{r_{i j}}-\frac{\pi}{m_{q}^{2}}\left[1+\frac{2}{3} \boldsymbol{\sigma}(i) \cdot \boldsymbol{\sigma}(j)\right] \delta\left(\mathbf{r}_{i j}\right)-\frac{3}{4 m_{q}^{2} r_{i j}^{3}} S(i, j)\right\} \\
\mathcal{V}_{\mathrm{con}}\left(\mathbf{r}_{i j}\right)=-a_{c} \boldsymbol{\lambda}(i) \cdot \boldsymbol{\lambda}(j) r_{i j}^{2} \\
\mathcal{V}_{\mathrm{OSE}}\left(\mathbf{r}_{i j}\right)=-\alpha_{\mathrm{ch}} \frac{4 m_{q}^{2}}{m_{\pi}^{2}} \frac{\Lambda_{\sigma}^{2}}{\Lambda_{\sigma}^{2}-m_{\sigma}^{2}} m_{\sigma}\left[\Upsilon\left(m_{\sigma} r_{i j}\right)-\frac{\Lambda_{\sigma}}{m_{\sigma}} \Upsilon\left(\Lambda_{\sigma} r_{i j}\right)\right] \\
\mathcal{V}_{\mathrm{OPE}}\left(\mathbf{r}_{i j}\right)=\frac{1}{3} \alpha_{\mathrm{ch}} \frac{\Lambda_{\pi}^{2}}{\Lambda_{\pi}^{2}-m_{\pi}^{2}} m_{\pi}\left\{\left[\Upsilon\left(m_{\pi} r_{i j}\right)-\frac{\Lambda_{\pi}^{3}}{m_{\pi}^{3}} \Upsilon\left(\Lambda_{\pi} r_{i j}\right)\right] \boldsymbol{\sigma}(i) \cdot \boldsymbol{\sigma}(j)\right. \\
\left.+\left[H\left(m_{\pi} r_{i j}\right)-\frac{\Lambda_{\pi}^{3}}{m_{\pi}^{3}} H\left(\Lambda_{\pi} r_{i j}\right)\right] S(i, j)\right\} \boldsymbol{\tau}(i) \cdot \boldsymbol{\tau}(j) .
\end{gathered}
$$

In Eqs. (2.2) the quark coordinates are denoted by $\mathbf{r}_{i}$ with the relative coordinate $\mathbf{r}_{i j}=\mathbf{r}_{i}-\mathbf{r}_{j}$, the operators $\boldsymbol{\sigma}(i), \boldsymbol{\tau}(i)$, and $\boldsymbol{\lambda}(i)$ are the spin, isospin, and eight-component color charge of quark $i$, respectively, and $S(i, j)$ is the quark tensor operator with

$$
\left.S(i, j)=3\left[\boldsymbol{\sigma}(i) \cdot \hat{\mathbf{r}}_{i j}\right] \boldsymbol{\sigma}(j) \cdot \hat{\mathbf{r}}_{i j}\right]-\boldsymbol{\sigma}(i) \cdot \boldsymbol{\sigma}(j) .
$$

The renormalized strong coupling constant is $\alpha_{s}, a_{c}$ regulates confinement, and $\alpha_{\mathrm{ch}}$ is chosen to reproduce the experimental pion-nucleon coupling constant, i.e.,

$$
\alpha_{\mathrm{ch}}=\left(\frac{3}{5}\right)^{2} \frac{g_{\pi N N}^{2}}{4 \pi} \frac{m_{\pi}^{2}}{4 m_{N}^{2}}=0.0288
$$

Chiral symmetry relates both meson coupling constants and it relates the regularizing cutoff masses $\Lambda_{\sigma}$ and $\Lambda_{\pi}$ by $\Lambda_{\sigma}=\Lambda_{\pi}$. The potential parameters are taken over from Refs. [7] and [8]; they are listed in Table I. Equations (2.2) use the standard Yukawa functions for $\Upsilon(x)$ and $H(x)$, i.e.,

TABLE I. Quark-model parameters. The parameters are taken from Refs. [7] and [8]; they are needed for the quark-quark potential of Eqs. (2.2). The single-quark wave functions in the nucleon and the $\Delta$ isobar are oscillator ground-state wave functions with oscillator parameter $b$.

\begin{tabular}{cc}
\hline \hline$m_{q}(\mathrm{MeV})$ & 313 \\
$b(\mathrm{fm})$ & 0.5 \\
\hline$\alpha_{s}$ & 0.4 \\
$a_{c}\left(\mathrm{MeV} \mathrm{fm}^{-2}\right)$ & 57.96 \\
$\alpha_{\mathrm{ch}}$ & 0.0288 \\
$m_{\sigma}\left(\mathrm{fm}^{-1}\right)$ & 3.42 \\
$\Lambda_{\sigma}\left(\mathrm{fm}^{-1}\right)$ & 4.2 \\
$m_{\pi}\left(\mathrm{fm}^{-1}\right)$ & 0.7 \\
$\Lambda_{\pi}\left(\mathrm{fm}^{-1}\right)$ & 4.2 \\
\hline \hline
\end{tabular}

$$
\begin{gathered}
\Upsilon(x)=\frac{e^{-x}}{x} \\
H(x)=\left(1+\frac{3}{x}+\frac{3}{x^{2}}\right) \Upsilon(x) .
\end{gathered}
$$

There are also contributions to the one-gluon and sigmameson exchanges which arise from the coupling of the quark spins to their orbital motion. Those contributions are small [9]; they are therefore left out in the present calculation and are not given in Eqs. (2.2a) and (2.2c).

The nucleon and $\Delta$ isobar are considered three-quark clusters, $\left|N \mathbf{r}_{N}\right\rangle$ and $\left|\Delta \mathbf{r}_{\Delta}\right\rangle$, each one bound to a center at $\mathbf{r}_{N}$ and $\mathbf{r}_{\Delta}$, respectively. The quark basis states

$$
\left|N \Delta \mathbf{r} S M_{S} T M_{T}\right\rangle=\mathcal{A}\left\{\left|N \frac{\mathbf{r}}{2}\right\rangle \otimes\left|\Delta-\frac{\mathbf{r}}{2}\right\rangle\right\}
$$

are tensor products of those cluster states, with the two centers separated by $\mathbf{r}$, their individual cluster spins and isospins coupled to a state $\left|S M_{S} T M_{T}\right\rangle$ of total spin $S$ and isospin $T$. The basis states are antisymmetrized, $\mathcal{A}$ being the quark antisymmetrizer. Nucleon- $\Delta$ partial waves with states $\left|N \Delta r(L S) I M_{I} T M_{T}\right\rangle$ are introduced, i.e.,

$$
\begin{aligned}
& \left|N \Delta \mathbf{r}(L S) I M_{I} T M_{T}\right\rangle \\
& =\sum_{M_{L} M_{S}} \int d^{2} \hat{\mathbf{r}}\left|N \Delta \mathrm{r} S M_{S} T M_{T}\right\rangle Y_{L M_{L}}(\hat{\mathbf{r}}) \\
& \times\left\langle L M_{L} S M_{S} \mid I M_{I}\right\rangle .
\end{aligned}
$$

They have definite angular momentum and isospin properties, $L$ being the orbital angular momentum of relative motion, $S$ the total spin, $I$ the total angular momentum, $M_{I}$ its projection, $T$ the total isospin, and $M_{T}$ its pro- 
jection. Those quark basis states are neither normalized at the same distance $r$, their distance-dependent norm being

$$
\begin{aligned}
& \mathcal{N}[r(L S) I T] \\
& \quad=\left\langle N \Delta r(L S) I M_{I} T M_{T} \mid N \Delta r(L S) I M_{I} T M_{T}\right\rangle
\end{aligned}
$$

nor orthogonal at different distances $r$; nor are they or- thogonal to states of different baryonic content at any distance $r$. In a configuration space representation, the basis states are functions of the quark coordinates $\mathbf{r}_{i}$.

The nucleon- $\Delta$ potential $V_{N \Delta}$ at relative distance $\mathbf{r}$ is identified with the interaction energy of the nucleon and $\Delta$-isobar clusters at cluster separation $\mathbf{r}$. In fact, the Born-Oppenheimer identification is done in partial waves. The nucleon- $\Delta$ potential is defined by

$$
\begin{aligned}
&\left\langle\mathbf{r}^{\prime}\left|V_{N \Delta}\right| \mathbf{r}\right\rangle=\sum_{L^{\prime} S^{\prime} I^{\prime} M_{I}^{\prime} T^{\prime} M_{T}^{\prime}} \int \rho^{\prime 2} d \rho^{\prime} \sum_{L S I M_{I} T M_{T}} \int \rho^{2} d \rho\left\langle\mathbf{r}^{\prime} \mid N \Delta \rho^{\prime}\left(L^{\prime} S^{\prime}\right) I^{\prime} M_{I}^{\prime} T^{\prime} M_{T}^{\prime}\right\rangle \\
& \\
& \times\left\langle N \Delta \rho^{\prime}\left(L^{\prime} S^{\prime}\right) I^{\prime} M_{I}^{\prime} T^{\prime} M_{T}^{\prime}\left|V_{N \Delta}\right| N \Delta \rho(L S) I M_{I} T M_{T}\right\rangle \\
& \times\left\langle N \Delta \rho(L S) I M_{I} T M_{T} \mid \mathbf{r}\right\rangle,
\end{aligned}
$$

with

$$
\begin{aligned}
&\left\langle N \Delta r^{\prime}\left(L S^{\prime}\right) I^{\prime} M_{I}^{\prime} T^{\prime} M_{T}^{\prime}\left|V_{N \Delta}\right| N \Delta r(L S) I M_{I} T M_{T}\right\rangle=\frac{\delta\left(r^{\prime}-r\right)}{r^{2}} \delta_{I^{\prime} I} \delta_{M_{I}^{\prime} M_{I}} \delta_{T^{\prime} T} \delta_{M_{T}^{\prime} M_{T}}\left\langle L^{\prime} S^{\prime}\left|V^{I T}(r)\right| L S\right\rangle \\
&\left\langle L^{\prime} S^{\prime}\left|V^{I T}(r)\right| L S\right\rangle= \frac{\left\langle N \Delta r\left(L^{\prime} S^{\prime}\right) I M_{I} T M_{T}\left|\mathcal{H}_{q}\right| N \Delta r(L S) I M_{I} T M_{T}\right\rangle}{\sqrt{\mathcal{N}\left[r\left(L^{\prime} S^{\prime}\right) I T\right]} \sqrt{\mathcal{N}[r(L S) I T]}} \\
&-\lim _{r \rightarrow \infty} \frac{\left\langle N \Delta r\left(L^{\prime} S^{\prime}\right) I M_{I} T M_{T}\left|\mathcal{H}_{q}\right| N \Delta r(L S) I M_{I} T M_{T}\right\rangle}{\sqrt{\mathcal{N}\left[r\left(L^{\prime} S^{\prime}\right) I T\right]} \sqrt{\mathcal{N}[r(L S) I T]}}
\end{aligned}
$$

By putting the distance-dependent norms $\mathcal{N}[r(L S) I T]$ into the denominators, the definition (2.6c) of the potential takes into account that the basis states are not normalized. The second term of Eq. (2.6c) takes out the internal quark energies of the separated nucleon- $\Delta$ system; it ensures the vanishing of the nucleon- $\Delta$ potential at large separations. Only the one-gluon and one-meson exchanges contribute [10]. The one-gluon-exchange part of the quark-quark potential makes its contribution when the two baryonic quark clusters overlap. The tail of the nucleon- $\Delta$ potential is dominated by sigma and pion exchange, both getting identical there to standard mesonexchange nucleon- $\Delta$ potentials.

References [7], [9], and [11] demonstrate that the quark Hamiltonian based on the potentials (2.2) yields a satisisfactory description of experimental properties in oneand two-baryon systems, when described in the framework of the resonating group method. Furthermore, the validity of the Born-Oppenheimer approximation is studied for the two-nucleon interaction in Refs. [12] and [13]. It is found to reflect the qualitative features of the twonucleon interaction as seen in a full resonating group treatment. Thus the approximation (2.6c) is also assumed to provide the qualitative characteristics of the nucleon- $\Delta$ potential and is therefore adopted in this paper.

In a quark description, the basis states $\left|N \Delta r(L S) I M_{I} T M_{T}\right\rangle$ are not orthonormalized as discussed. However, in the subsequent baryonic description of hadronic reactions in Secs. III and IV, the same states are assumed to be properly orthonormalized. For example the baryonic nucleon- $\Delta$ potential of Eq. (2.6b) is defined between nonorthonormalized quark states, but is assumed to act between corresponding orthonormalized baryonic basis states.

\section{B. Potential based on meson exchange}

References [14] and [15] employ a nucleon- $\Delta$ potential $V_{N \Delta}$ derived from meson exchange. The mesons considered there are the pion, sigma, rho, and omega. All mesons contribute to the direct potential $V_{N \Delta}^{d}$; only the pion and rho mesons also contribute to its exchange part $V_{N \Delta}^{e}$. The potential acts in states of isospin $T=1$ and 2; only its isotriplet $T=1$ states couple to those of the two-nucleon and pion-deuteron systems. In nonrelativistic approximation of lowest order, the nucleon- $\Delta$ potential becomes local in configuration space and takes the form

$$
\begin{aligned}
\left\langle\mathbf{r}^{\prime}\left|V_{N \Delta}\right| \mathbf{r}\right\rangle= & \delta\left(\mathbf{r}^{\prime}-\mathbf{r}\right)\left[V_{N \Delta}^{d}(\mathbf{r})+V_{N \Delta}^{e}(\mathbf{r})\right] \\
V_{N \Delta}^{d}(\mathbf{r})= & {\left[1+P_{12}\right] \tau_{N N}(1) \cdot \tau_{\Delta \Delta}(2) } \\
& \times\left[V_{C}^{d}(r)+V_{\sigma}^{d}(r) \sigma_{N N}(1) \cdot \sigma_{\Delta \Delta}(2)\right. \\
& \left.+V_{T}^{d}(r) S_{N \Delta}^{N \Delta}(1,2)\right] \\
V_{N \Delta}^{e}(\mathbf{r})= & {\left[1+P_{12}\right] \tau_{N \Delta}(1) \cdot \tau_{N \Delta}^{\dagger}(2) } \\
& \times\left[V_{\sigma}^{e}(r) \sigma_{N \Delta}(1) \cdot \sigma_{N \Delta}(2)\right. \\
& \left.+V_{T}^{e}(r) S_{N \Delta}^{\Delta N}(1,2)\right]
\end{aligned}
$$

In Eqs. (2.8), $\mathbf{r}$ is the relative coordinate, pointing from baryon 2 to baryon $1 ; P_{12}$ interchanges the baryons 1 and 2. Furthermore, $\sigma_{N N}(i)\left[\tau_{N N}(i)\right]$ is the spin 
[isospin] operator of nucleon $i, \sigma_{N \Delta}(i)\left[\tau_{N \Delta}(i)\right]$ denotes the transition spin [isospin] from nucleon $i$ to a $\Delta$ isobar, and $\sigma_{\Delta \Delta}(i)\left[\tau_{\Delta \Delta}(i)\right]$ is the spin [isospin] operator when baryon $i$ is a $\Delta$ isobar; the respective reduced spin [isospin] matrix elements are $\sqrt{6}, 2$, and $2 \sqrt{15}$. The direct and exchange nucleon- $\Delta$ tensor operators $S_{N \Delta}^{N \Delta}(1,2)$ and $S_{N \Delta}^{\Delta N}(1,2)$ are defined as

$$
\begin{aligned}
S_{N \Delta}^{N \Delta}(1,2)= & 3\left[\sigma_{N N}(1) \cdot \hat{\mathbf{r}}\right]\left[\sigma_{\Delta \Delta}(2) \cdot \hat{\mathbf{r}}\right] \\
& -\sigma_{N N}(1) \cdot \sigma_{\Delta \Delta}(2), \\
S_{N \Delta}^{\Delta N}(1,2)= & 3\left[\sigma_{\Delta N}(1) \cdot \hat{\mathbf{r}}\right]\left[\sigma_{\Delta N}^{\dagger}(2) \cdot \hat{\mathbf{r}}\right] \\
& -\sigma_{\Delta N}(1) \cdot \sigma_{\Delta N}^{\dagger}(2) .
\end{aligned}
$$

The meson coupling constants and the regularizing cutoff masses of the hadronic form factors required for the radial shapes of the potentials $V_{N \Delta}^{d}(\mathbf{r})$ and $V_{N \Delta}^{e}(\mathbf{r})$ are not made explicit in Eqs. (2.8). Those parameters are experimentally unknown. The coupling constants between mesons and $\Delta$ isobar are scaled from the corresponding nucleonic ones by means of a naive $\mathrm{SU}(2)$ quark model $[16]$, except for the pion-nucleon- $\Delta$ case. All potential parameters are those of Table II in Ref. [15], with further explanations given in Ref. [14]. The exchange of the sigma, rho, and omega mesons also yields a spin-orbit part in the direct potential $V_{N \Delta}^{d}(\mathbf{r})$. That part was left out in the calculations of Refs. [14,15]; it will be left out, for reasons of consistency with the quark-model-based potential, in the present calculation as well as is therefore not included in the potential (2.8).

In applications, the nucleon- $\Delta$ potential is used in a partial-wave projected form, corresponding to the form of Eq. (2.6b).

\section{Comparison of the two different $N-\Delta$ potentials}

The nucleon- $\Delta$ potentials are compared in configuration space. Characteristic features are the following: The potential derived from nonrelativistic quark dynamics is rather smooth; at relative distances smaller than $1 \mathrm{fm}$, it becomes strongly repulsive in most partial waves; the coupling between partial waves connected by the tensor part of the potential is weak. In contrast, the potential based on meson exchange has more structure; in particular, a remarkable attractive pocket at small relative distances exists in most partial waves; the tensor coupling is strong. Both potentials become almost identical outside $2 \mathrm{fm}$ : However, the pion-nucleon- $\Delta$ coupling strength employed in the meson-exchange potential [14] is derived from $\Delta$ decay and therefore differs form the one effectively arising in the quark-model potential; thus, the pion-exchange parts of the two potentials cannot become fully the same in their tails. Furthermore, though the sigma-meson coupling constant is almost the same in both potential models, the sigma mass, the cutoff mass, and the form of the regularization are so different that the two potential models still remain distinct at distances between 1.5 and $2 \mathrm{fm}$. The configuration-space comparison between the two potentials is carried out in Fig. 1 for some important isotriplet partial waves, i.e., ${ }^{3} S_{1},{ }^{5} S_{2}$, and ${ }^{5} P_{3}$. That comparison confirms the general characteristics of the potentials.

The found pronounced short-range repulsion of the (a)

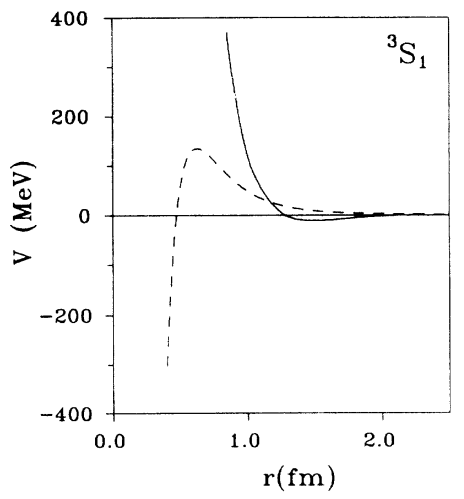

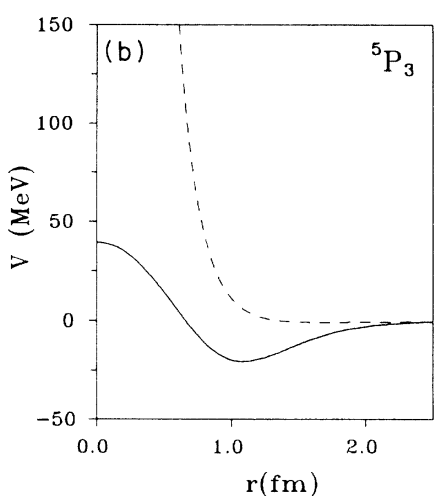

FIG. 1. Nucleon- $\Delta$ potential $V_{N} \Delta$ in the isotriplet partial waves ${ }^{3} S_{1},{ }^{5} S_{2}$, and ${ }^{5} P_{3}$ as function of relative distance $r$ between baryons. Those partial waves are coupled ones. The channel-diagonal potentials are shown, except for the tensor-coupled potential ${ }^{5} S_{2^{-}}{ }^{5} D_{2}$, which is also given. The solid curve refers to the quark-model nucleon- $\Delta$ potential of this paper, the dashed curve to the meson-exchange nucleon- $\Delta$ potential of Refs. [14,15]. 

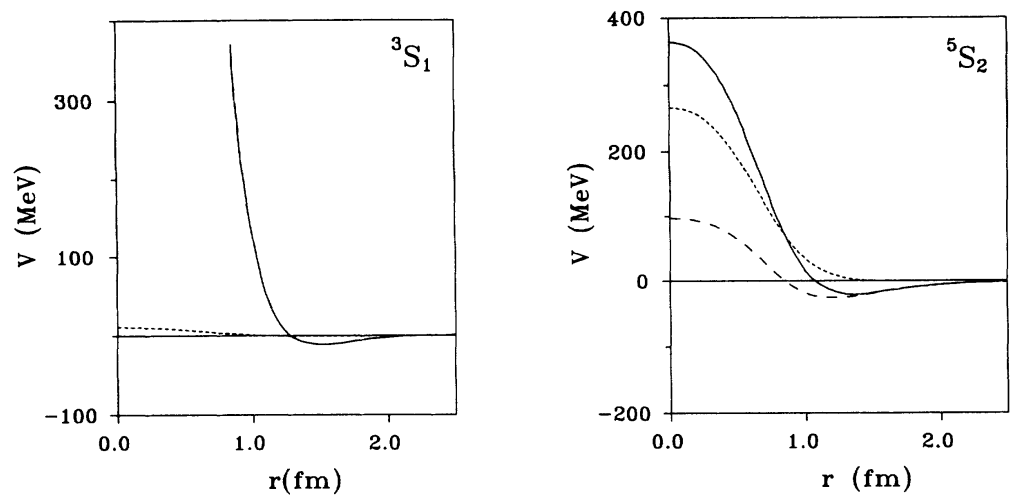

FIG. 2. Quark-model nucleon- $\Delta$ potential $V_{N \Delta}$ as function of the relative distance $r$. Contributions to the channel-diagonal potentials in the isotriplets ${ }^{3} S_{1}$ and ${ }^{5} S_{2}$ partial waves are displayed. The dotted curve refers to the one-gluon-exchange contribution, the dashed curve to the meson-exchange quark-quark contribution; the solid curve is the full result. In the ${ }^{3} S_{1}$ potential, the full results and its meson-exchange contribution are graphically indistinguishable. quark-model nucleon- $\Delta$ potential is consistent with previous work: Six-quark states can be classified by irreducible representations of the spin-isospin $\mathrm{SU}(4)$ group. Only its $\{5,1\}$ orbital symmetry occurs in the $S=T=1$ and $S=T=2$ partial waves. References [12] and [17] demonstrate that at zero relative distance the spinisospin $\{5,1\}$ symmetry can just be combined with the orbital $[4,2]$ symmetry to a totally antisymmetric quark state, the totally symmetric [6] symmetry being forbidden. In the resonating group description of two-nucleon scattering, that forbidden state of orbital [6] symmetry produces a node in the relative two-nucleon wave function according to Ref. [18] and that node effectively yields hard-core-type two-nucleon phase shifts. The description of two-nucleon scattering in terms of local BornOppenheimer potentials corresponding to Eq. (2.6c) simulates the same short-range repulsion in a different way: In those partial waves in which the forbidden state occurs, the normalization $\mathcal{N}[r(L S) I T]$, included in the definition (2.6c) of the potentials, vanishes at small relative distances as $r^{4}$, making the potential repulsive at short distances and even singular at $r=0$; when expanding the quark-quark potentials in terms of Gaussian for computational convenience, that singularity gets regularized, though the short-range repulsion remains qualitatively untouched. Returning to the nucleon- $\Delta$ potential of Eq. (2.6), the short-range repulsion in partial waves with forbidden orbital [6] symmetry does not arise dominantly from the one-gluon exchange, whose matrix elements vanish in the same way as the wave function overlap. Instead, the short-range repulsion arises mostly from the meson
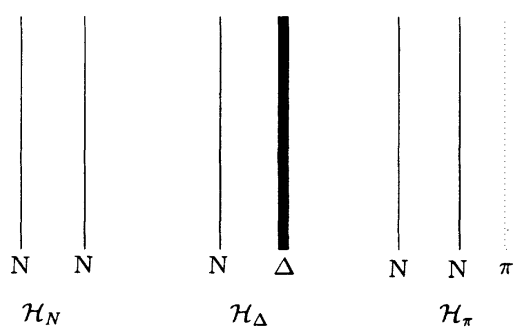

FIG. 3. Hilbert space of baryon number 2 for the considered force model. Besides the purely nucleonic sector $\mathcal{H}_{N}$, there is a second sector $\mathcal{H}_{\Delta}$ in which one nucleon is turned into a $\Delta$ isobar and a third sector $\mathcal{H}_{\pi}$ in which a pion is added as third particle. exchange between quarks, whose matrix elements vamish with a smaller power in $r$ than the wave function overlap. Thus the meson-exchange contribution is enhanced by the decreasing overlap, according to the definition (2.6c) of the nucleon- $\Delta$ potential. That described source for repulsion is illustrated in Fig. 2. In contrast, in partial waves in which the totally symmetric orbital symmetry [6] is not forbidden at zero relative distances, e.g., in the isotriplet ${ }^{5} S_{2}$ partial wave, the repulsion is dominated by gluon exchange.

Phenomenologically, such a short-range repulsion is supported: The theoretical analysis of elastic piondeuteron scattering by Ferreira and Dosch [19] suggests a strong short-range repulsion for the nucleon- $\Delta$ inter-

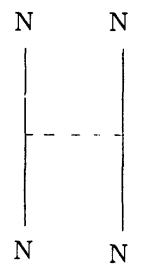

(a)

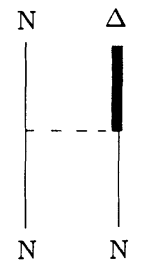

(b)

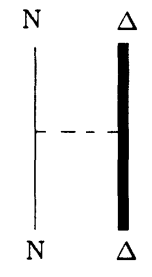

(c)

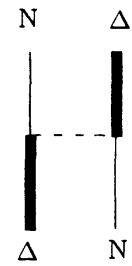

(d)

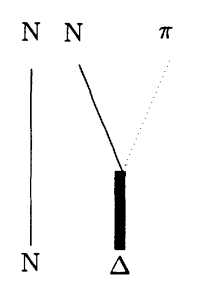

(e)

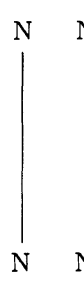

(f)

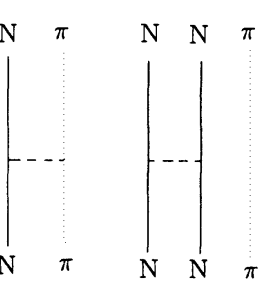

(g)
FIG. 4. Building blocks of the interaction $H_{1}$ in the considered force model. They are given in the Hilbert space of baryon number 2. The dashed horizontal lines denote instantaneous potentials. Processes (c) and (d) are the direct and exchange nucleon- $\Delta$ potentials whose effects on the two-nucleon system above the pion threshold are studied in this paper. Process (e) is of one-baryon nature; it is assumed to yield the full pion-nucleon interaction in the $P_{33}$ partial wave by iteration. Process (f) stands for the pion-nucleon potential in partial waves other than $P_{33}$. All parts of the interaction contribute to isotriplet partial waves. In isosinglet partial waves, the interaction reduces to process (a). 
action in the ${ }^{3} S_{1}$ wave. Furthermore, there is evidence for such a repulsion from the description of inelastic pion scattering off ${ }^{12} \mathrm{C}$ within the framework of the $\Delta$-hole model [20].

The meson-exchange nucleon- $\Delta$ potential is dominated by pion and sigma exchange at large relative distances. At small relative distances, all mesons contribute with weights which depend on the partial wave. The somehow unsmooth behavior of the potential there, e.g., the attractive pocket in the isotriplet partial waves ${ }^{3} S_{1}$ and ${ }^{5} S_{2}$ of Fig. 1, is a remainder of the $\delta$-function contributions to the spin-dependent parts $V_{\sigma}^{d}(r)$ and $V_{\sigma}^{e}(r)$ of the potential which are regularized with large cutoff masses. In case the meson exchanges were regularized by taking out the $\delta$-function contributions entirely, the meson-exchange nucleon- $\Delta$ potential would show repulsion at small relative distances in the isotriplet partial waves ${ }^{3} S_{1}$ and ${ }^{5} S_{2}$ in qualitatively the same way as the quark model does.

\section{CALCULATIONAL FRAMEWORK FOR THE DESCRIPTION OF HADRONIC REACTIONS}

This paper calculates observables of elastic and inelastic nucleon-nucleon and pion-deuteron scattering. The considered reactions are theoretically described by a Hamiltonian $\mathrm{H}=\mathrm{H}_{0}+\mathrm{H}_{1}$ in a Hilbert space in which the non-nucleonic $\Delta$ isobar and pion degrees of freedom are added to the nucleonic one. The considered sectors of the Hilbert space are shown in Fig. 3 for baryon number 2 ; the projector onto the purely baryonic sector is denoted by $P_{N}$, the one onto the sector with a $\Delta$ isobar by $P_{\Delta}$, and the one onto the sector with a pion by $Q$. The Hamiltonian acting in that Hilbert space has the ki-

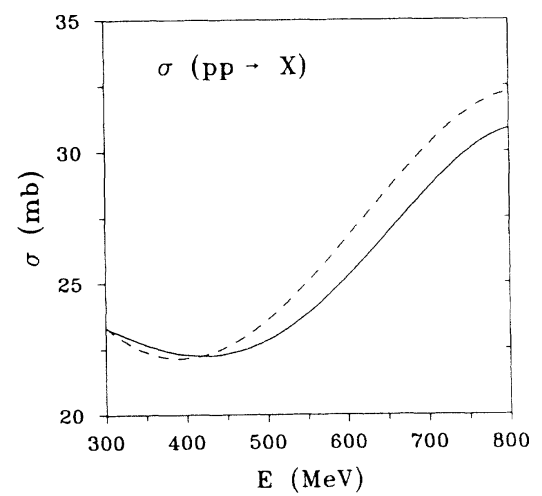

FIG. 5. Total proton-proton cross section $\sigma(p p \rightarrow X)$ as function of the proton laboratory kinetic energy $E$. The effect of different identifications between experimental and calculational kinematics is indicated. The solid curve refers to the identification used in this paper: The c.m. energy is relativistically evaluated from the experimental laboratory energy and used for the calculation of the on-shell multichannel transition matrix. The dashed curve uses the alternative identification of Ref. [23]. The c.m. energy is nonrelativistically evaluated from the experimental laboratory energy and used for the calculation of the on-shell multichannel transition matrix

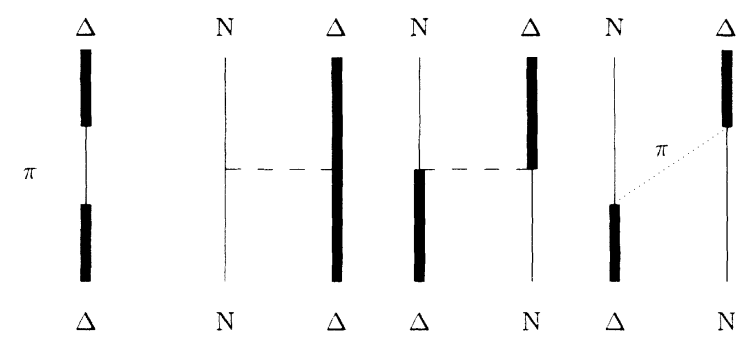

$\begin{array}{llll}\text { (a) } & \text { (b) } & \text { (c) } & \text { (d) }\end{array}$

FIG. 6. Effective hadronic interaction in the Hilbert sector with a $\Delta$ isobar. The energy-dependent contributions arising from the explicit propagation of a pion are of one-baryon nature, i.e., process (a) yielding $P_{\Delta} \delta H_{0}(z) P_{\Delta}$, and of two-baryon nature, i.e., process (d) yielding $P_{\Delta} \delta H_{1}(z) P_{\Delta}$; the simplification $Q H_{1} Q=0$ is used. The instantaneous potentials (b) and (c) are irreducible building blocks of the employed force model defined diagrammatically in Fig. 4

netic energy part $H_{0}$ and the interaction part $H_{1}$. The building blocks of the interaction are displayed in Fig. 4; they can couple the different sectors of the Hilbert space. The description of the considered reactions uses the Alt-Grassberger-Sandhas [21] three-particle scattering theory extended to accommodate particle absorption [22]. The technical apparatus of Ref. [15] is taken over without essential changes for the calculation.

Kinematically, relativistic expressions are used for the observables. In contrast, the one-shell multichannel tran-

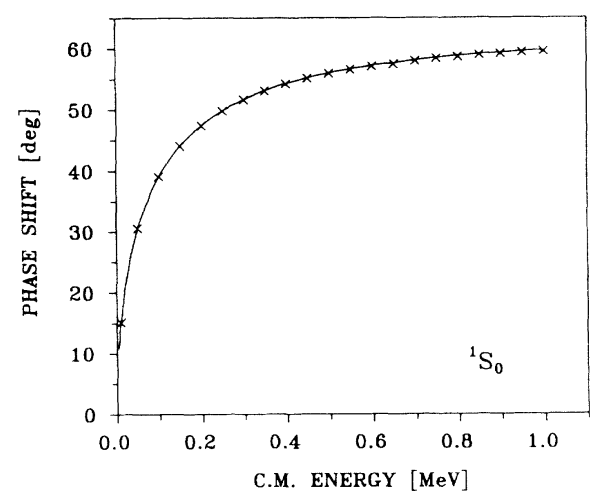

FIG. 7. Low-energy ${ }^{1} S_{0}$ two-nucleon phase shifts as function of the nucleon c.m. energy. The solid curve refers to the force model of Fig. 4, the nucleon- $\Delta$ potential being the meson-exchange one in the identification (3.2b), thus slightly changed compared with Ref. [15]; the effective-range parameters are $a=-17.53 \mathrm{fm}$ and $r_{0}=2.84 \mathrm{fm}$. The crosses refer to the Paris potential, whose effective-range parameters are $a=-17.53 \mathrm{fm}$ and $r_{0}=2.88 \mathrm{fm}$. Coulomb-subtracted phase shifts are shown. A comparison to experimental data is omitted, since it would require the inclusion of the Coulomb interaction in the calculation of the phase shifts. The force model of Ref. [15] does not define the nucleon- $\Delta$ potential according to Eq. (3.1) and therefore does not show exact phase equivalence at zero kinetic energy. The phase nonequivalence would not show up as sizable in the figure; however, the effective-range parameters are with $a=-20.04 \mathrm{fm}$ and $r_{0}=2.80$ fm dramatically different. 
sition matrix required for calculating the observables is derived with a half-relativistic form of the kinetic energy operator $H_{0}$, i.e., the baryons being treated nonrelativistically, the pion relativistically: The experimental c.m. energy of a reaction is evaluated relativistically from the laboratory energy and then taken as the available energy for the transition matrix of the calculation, for which the on-shell momenta are theoretically determined from that energy according to half-relativistic kinematics; in that way the employed transition matrix becomes on shell in an internally consistent form. That kinematic identification between experimental kinematics and theoretical description is indeed a nonunique recipe. A variety of alternative recipes is possible, and in nucleon-nucleon scattering the theoretical predictions depend on that recipe, as Fig. 5 demonstrates for the total cross section: The solid curve follows the identification adopted in this paper. For the results given by the dashed curve, the c.m. energy used in the calculation is related nonrelativistically to the experimental laboratory energy as in Ref. [23]. The dependence of results on the kinematic identification between experimental and theoretical descriptions is most severe in nucleon-nucleon scattering.
Dynamically, the choice of the interaction $H_{1}$ in the force model of Fig. 4 also follows mostly Ref. [15].

(1) The pion-nucleon interaction is assumed to proceed exclusively in the $P_{33}$ partial wave, being mediated solely by the excitation of the $\Delta$ isobar according to Fig. 4(e). The pion-nucleon potential in all other partial waves, i.e., process (f) of Fig. 4, is neglected. Furthermore, also the nucleon-nucleon potential in the presence of a pion, i.e., process (g) of Fig. 4, is left out. Thus the interaction in the Hilbert sector with a pion is assumed to vanish altogether, i.e., $Q H_{1} Q=0$. In the description of the considered reactions, baryonic transition matrices are computed as intermediate technical quantities to which the propagation in the pionic sector contributes effective interaction corrections of one-baryon and two-baryon nature, i.e., $P_{\Delta} \delta H_{0}(z) P_{\Delta}$ and $P_{\Delta} \delta H_{1}(z) P_{\Delta}$, respectively, $z$ being the energy available for the respective processes. Those corrections only occur in the Hilbert sector with a $\Delta$ isobar. They are illustrated as processes (a) and (d) in Fig. 6; they are given there already specialized to the adopted assumption $Q H_{1} Q=0$.

(2) The instantaneous nucleon-nucleon potential
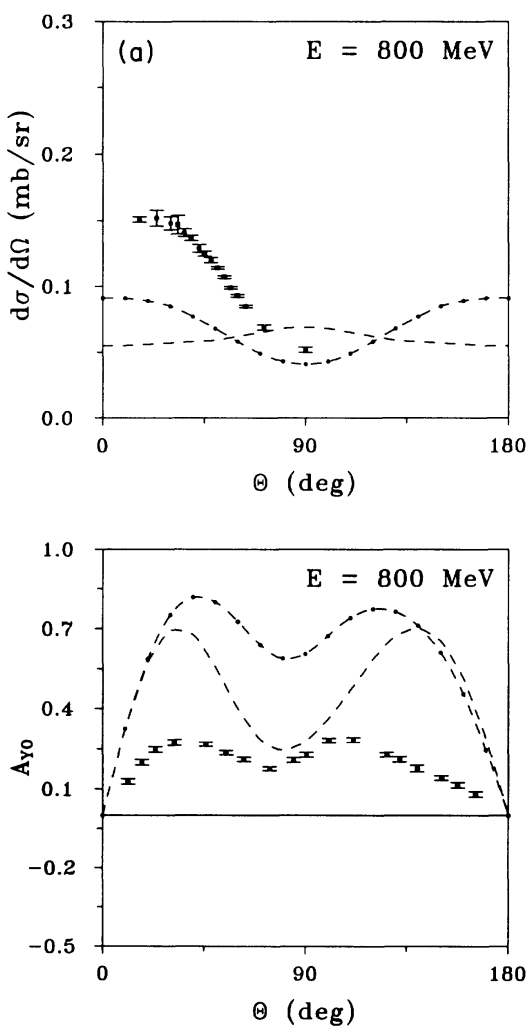
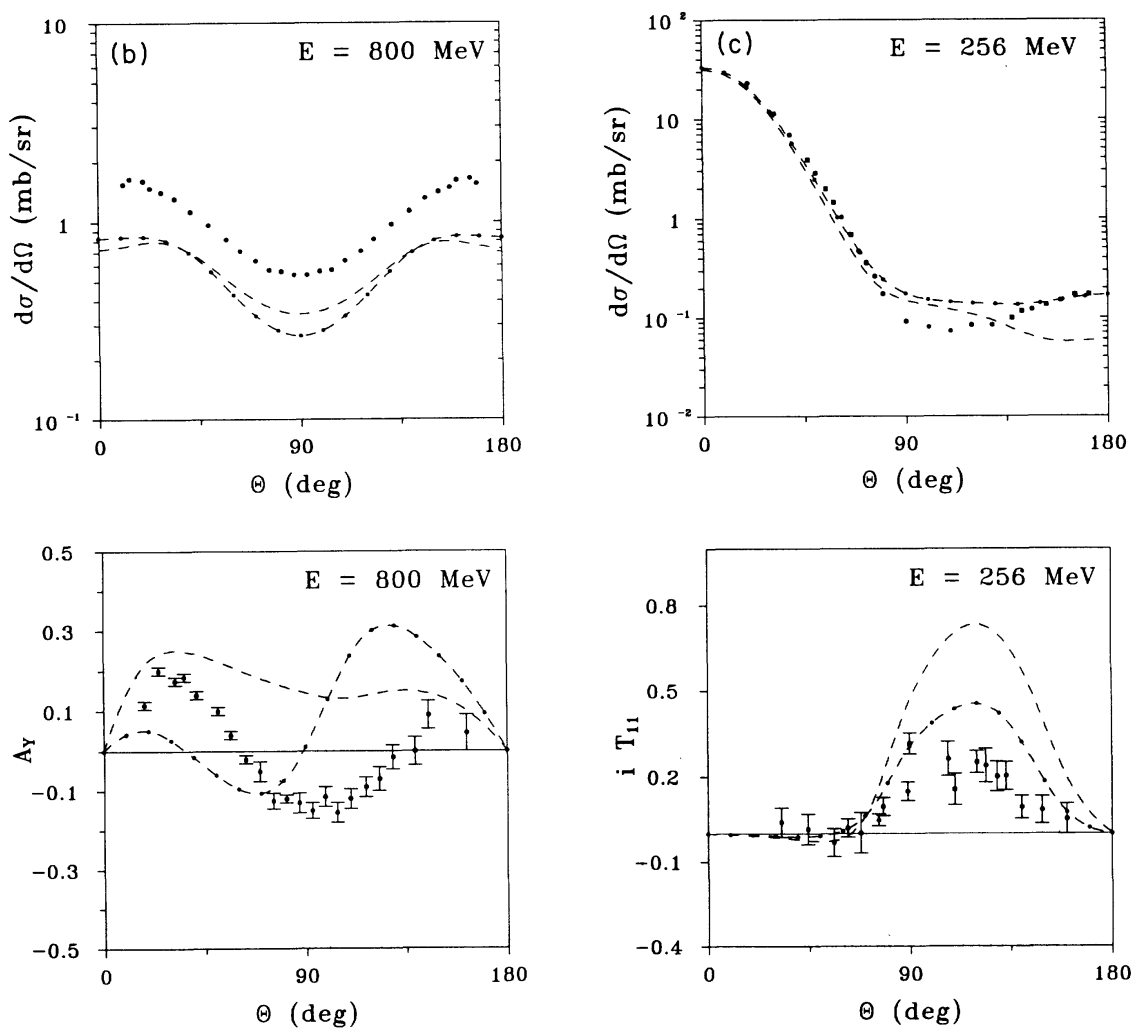

FIG. 8. Comparison of results based on the meson-exchange nucleon- $\Delta$ potential with the two different identifications (3.2b) and (3.2c) for the pion-exchange part. Especially sensitive observables of the considered reactions are displayed, i.e., the differential cross section $d \sigma / d \Omega$ and the analyzing power $A_{y 0}$ for the reaction $p p \rightarrow \pi^{+} d$ at $800 \mathrm{MeV}$ proton laboratory kinetic energy in (a), the differential cross section $d \sigma / d \Omega$ and the beam asymmetry $A_{y}$ for the reaction $p p \rightarrow n \Delta^{++}\left(p \pi^{+}\right)$at $800 \mathrm{MeV}$ proton laboratory kinetic energy in (b), and the differential cross section $d \sigma / d \Omega$ and the vector polarization $i T_{11}$ for elastic pion-deuteron scattering at $256 \mathrm{MeV}$ pion laboratory kinetic energy in (c). The dashed curve refers to the results obtained from the meson-exchange nucleon- $\Delta$ potential with the pion-exchange part according to Eq. (3.2b) as used throughout this paper, the dash-dotted curve to those with the pion-exchange part according to Eq. (3.2c) as used in Ref. [15]. The data are the same as used later on in Figs. 14-16. 


$$
P_{N} H_{1} P_{N}=V_{N N}-P_{N} H_{1} P_{\Delta} \frac{P_{\Delta}}{2 m_{N}-P_{\Delta}\left[H_{0}+\delta H_{0}\left(2 m_{N}\right)+H_{1}+\delta H_{1}\left(2 m_{N}\right)\right] P_{\Delta}} P_{\Delta} H_{1} P_{N}
$$

is slightly changed compared to the definition (2.24) of Ref. [15]. The pionic corrections $P_{\Delta} \delta H_{0}(z) P_{\Delta}$ and $P_{\Delta} \delta H_{1}(z) P_{\Delta}$ in the two-baryon system are included in that changed definition at zero kinetic two-nucleon energy $2 m_{N}$. The definition (3.1) guarantees exact phase equivalence with the nucleonic reference potential $V_{N N}$, here taken to be the Paris potential [24], at zero kinetic energy, and substantially improves the approximate phase equivalence at small kinetic energies of elastic nucleon-nucleon scattering. That fact is borne out in Fig. 7. The improvement (3.1) for the two-nucleon part of the force model is conceptually important; however, for all the observables reported in Sec. IV is at most in the fourth significant digit and can therefore not been seen in any plot.

(3) This paper studies the effect of the instantaneous nucleon- $\Delta$ potential $P_{\Delta} H_{1} P_{\Delta}$ on the properties of the two-nucleon system above the pion threshold. However, even without such an instantaneous potential, there is an effective energy-dependent interaction $P_{\Delta} \delta H_{1}(z) P_{\Delta}$ mediated by the exchange of an explicit pion between $\Delta$ isobars. The complete two-body nucleon- $\Delta$ interaction $P_{\Delta}\left[H_{1}+\delta H_{1}(z)\right] P_{\Delta}$ is displayed in Fig. 6 by the processes (b)-(d). Process (c) of Fig. 6 should not contain that time ordering of pion exchange any longer, which process (d) accounts for in an explicit and retarded fashion. The effects of different parametrizations of the nucleon- $\Delta$ potential are studied.

(3a) In a reference calculation, only the pion-mediated exchange interaction is kept, however, with both its time orderings. Since process (d) of Fig. 6 yields one time ordering only, the other time ordering is to be added as an instantaneous potential in process (c), its particular form chosen as the energy-independent reduction of process (d), i.e.,

$$
P_{\Delta}\left[H_{1}+\delta H_{1}(z)\right] P_{\Delta}=P_{\Delta} \delta H_{1}\left(z_{o n}\right) P_{\Delta}+P_{\Delta} \delta H_{1}(z) P_{\Delta},
$$

with $z_{o n}=2 m_{N}+\left(p^{2}+{p^{\prime}}^{2}\right) / 2 m_{N}, \mathbf{p}$ and $\mathbf{p}^{\prime}$ being the relative nucleon- $\Delta$ momenta between which the matrix element is to be computed.

(3b) In the calculation of this paper, a full instantaneous nucleon- $\Delta$ potential is employed. The pionic contribution to the direct and exchange parts of the nucleon$\Delta$ potential $V_{N \Delta}$, shown as processes (b) and (c) in Fig. 6 and derived from nonrelativistic quark dynamics, could in principle be isolated; in practice, that isolation of terms is not done. Thus the contribution (d) in Fig. 6 of explicit pion exchange has to be extracted from $V_{N \Delta}$ in an energy-averaged form, in order to avoid overcounting that particular process, i.e.,

$$
\begin{aligned}
P_{\Delta}\left[H_{1}\right. & \left.+\delta H_{1}(z)\right] P_{\Delta} \\
& =\left[V_{N \Delta}-P_{\Delta} \delta H_{1}\left(z_{o n}\right) P_{\Delta}\right]+P_{\Delta} \delta H_{1}(z) P_{\Delta}
\end{aligned}
$$

where $z_{\text {on }}$ is chosen as for Eq. (3.2a).

(3c) If the pionic contribution $V_{N \Delta}^{e}[\pi]$ to the exchange part of the nucleon- $\Delta$ potential in Fig. $4(\mathrm{~d})$ could easily be extracted from the full potential, as is the case for the meson-exchange nucleon- $\Delta$ potential of Sec. II B, the alternative choice

$$
\begin{aligned}
P_{\Delta}\left[H_{1}+\delta H_{1}(z)\right] P_{\Delta} & \\
= & {\left[V_{N \Delta}-V_{N \Delta}^{e}[\pi]+P_{\Delta} \delta H_{1}\left(z_{o n}\right) P_{\Delta}\right] } \\
& +P_{\Delta} \delta H_{1}(z) P_{\Delta}
\end{aligned}
$$

is possible. That form of the nucleon- $\Delta$ interaction appeared to Ref. [15] especially appropriate for a comparison with the reference calculation of Eq. (3.2a). However, its use in this paper for the meson-exchange nucleon- $\Delta$ potential would introduce an unwanted asymmetry into the comparison with the nucleon- $\Delta$ potential derived from nonrelativistic quark dynamics for which the definition (3.2b) has to be taken. Thus this paper will
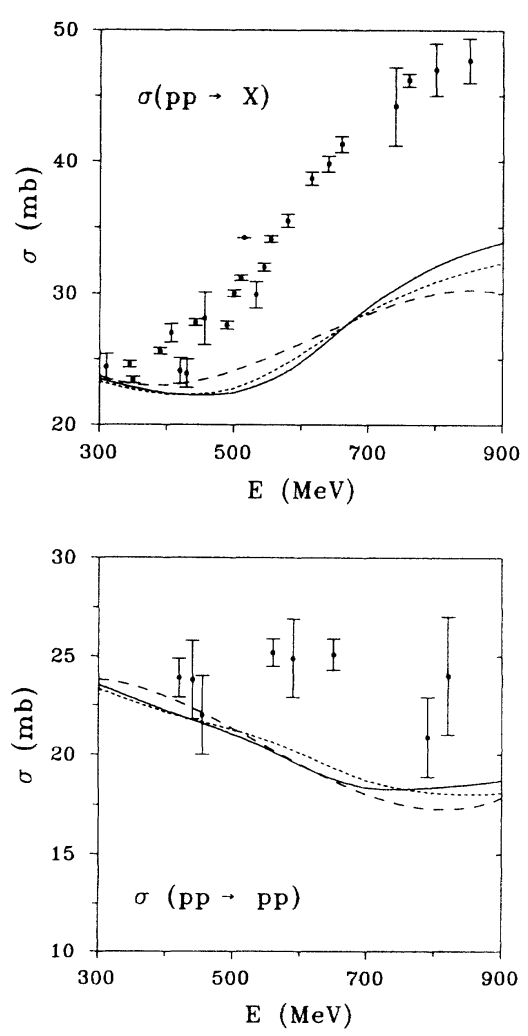

FIG. 9. Total proton-proton cross sections as function of the proton laboratory kinetic energy. The solid curve refers to the results obtained with the quark-model nucleon- $\Delta$ potential, the dashed curve to those with the meson-exchange nucleon- $\Delta$ potential. The dotted curve is the reference calculation without a nucleon- $\Delta$ potential according to Eq. (3.2a). The data are taken from Ref. [25]. 

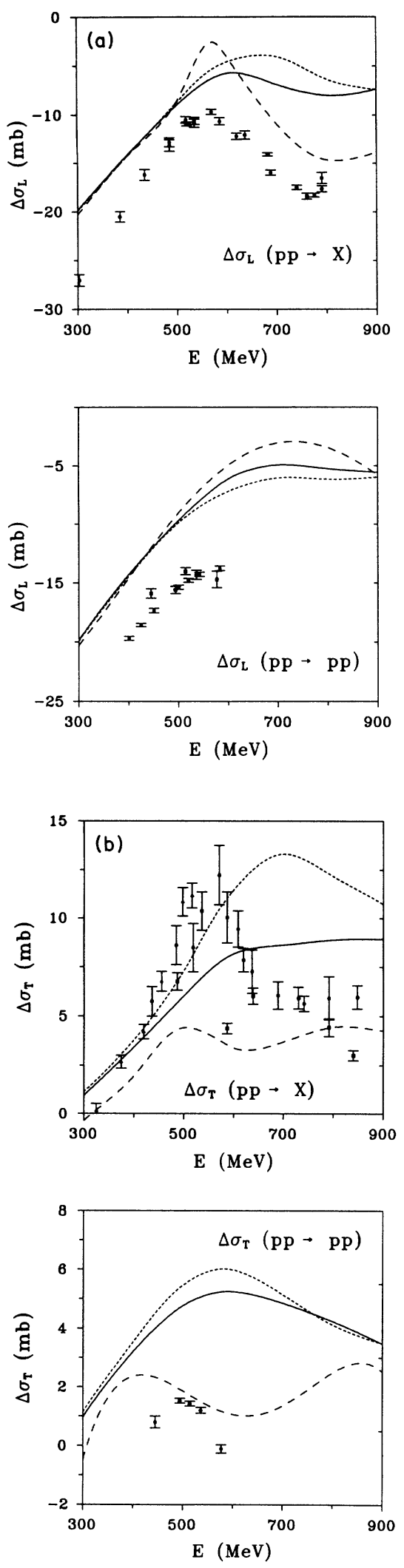

FIG. 10. Total proton-proton polarization cross sections $\Delta \sigma_{L}=\sigma(\rightleftarrows)-\sigma(\rightrightarrows)$ and $\Delta \sigma_{T}=\sigma(\uparrow \downarrow)-\sigma(\uparrow \uparrow)$ as function of the proton laboratory kinetic energy. The curves have the same meaning as in Fig. 9. The data are taken from Ref. [26].

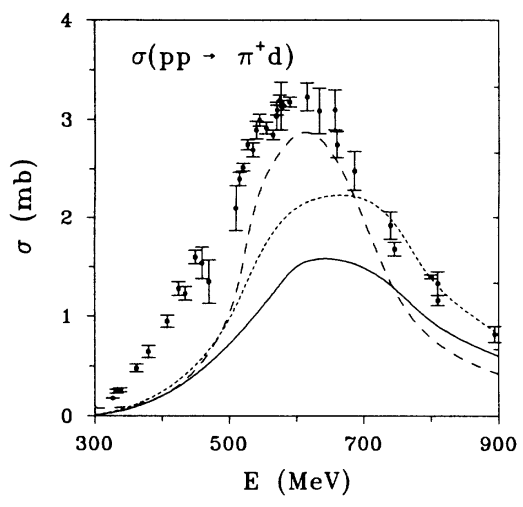

FIG. 11. Total cross section for the reaction $p p \rightarrow \pi^{+} d$ as function of the proton laboratory kinetic energy $E$. The curves have the same meaning as in Fig. 9. The data are taken from the compilation of Ref. [27] and from Ref. [28].

use the prescription (3.2b) also for the meson-exchange nucleon- $\Delta$ potential in contrast to Ref. [15], which used prescription (3.2c).

All other building blocks of the force model defined in Fig. 4 are employed as in Ref. [15]. Observables of elastic and inelastic nucleon-nucleon and pion-deuteron scattering are calculated for the force model of Fig. 4 with the instantaneous nucleon- $\Delta$ potential derived from nonrelativistic quark dynamics. The predictions are compared to those based on a meson-exchange nucleon- $\Delta$ potential,
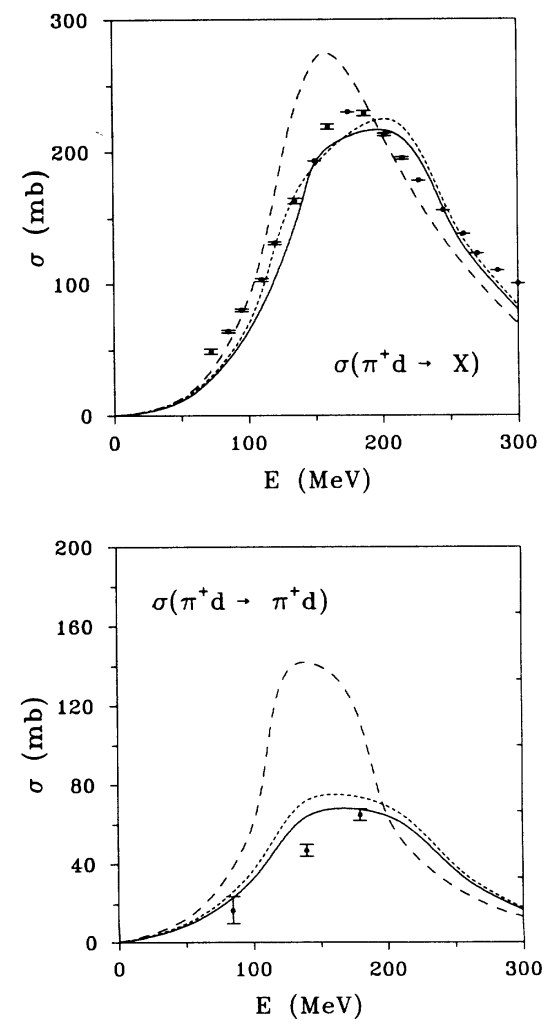

FIG. 12. Total pion-deuteron cross sections as function of the pion laboratory kinetic energy $E$. The curves have the same meaning as in Fig. 9. The data are taken from Refs. [28] and [29]. 
the latter results already given in Ref. [15]. Though the parameters of the meson-exchange potential are taken over unchanged from Ref. [15], its identification with the instantaneous nucleon- $\Delta$ potential $P_{\Delta} H_{1} P_{\Delta}$ of the employed force model has been changed: This paper uses the identification (3.2b), whereas Ref. [15] uses the identification (3.2c). That adjustment, done for the case of comparison with the quark-model-based potential, appears absolutely minor; however, it turns out to create substantial variations in the computed observables. Examples in which the sensitivity is maximal are shown in Fig. 8; the examples also prove that the pion contribution in the meson-exchange nucleon- $\Delta$ potential is most important. Though the two potential contributions $\frac{1}{2} V_{N \Delta}^{e}[\pi]$ and $P_{\Delta} \delta H_{1}\left(z_{o n}\right) P_{\Delta}$ describe the same physical process, i.e., one time ordering of pion exchange between a nucleon and a $\Delta$ isobar, they describe it in a technically different fashion: The contribution $P_{\Delta} \delta H_{1}\left(z_{o n}\right) P_{\Delta}$ is an energy average of process (d) in Fig. 6 and works with very small regularizing masses in the pion vertices; it is therefore a very smooth potential at intermediate momenta. The contribution $\frac{1}{2} V_{N \Delta}^{e}[\pi]$ uses the large cutoff masses of the full meson-exchange nucleon- $\Delta$ potential; it is therefore not suppressed at all at intermediate energies. Thus the combination $V_{N \Delta}^{e}[\pi]-P_{\Delta} \delta H_{1}\left(z_{o n}\right) P_{\Delta}$ and the term $P_{\Delta} \delta H_{1}\left(z_{o n}\right) P_{\Delta}$ are quite different and their difference creates the changes in the physics results, which the two different identifications (3.2b) and (3.2c) of the instantaneous part $P_{\Delta} H_{1} P_{\Delta}$ in the nucleon- $\Delta$ interaction with the nucleon- $\Delta$ potential $V_{N \Delta}$ yield and which are illustrated in Fig. 8.

\section{RESULTS AND DISCUSSION}

Observables of elastic and inelastic nucleon-nucleon and pion-deuteron scattering are calculated. The results refer to total cross sections and spin-averaged and spindependent differential cross sections. All figures of this section are composed in the same way: The results obtained with the quark-model nucleon- $\Delta$ potential are always shown as solid curves and those with the mesonexchange nucleon- $\Delta$ potential as dashed curves; reference results, which are derived without a proper nucleon- $\Delta$ potential, only including a pion-exchange piece according to the identification (3.2a), are given by dotted lines;
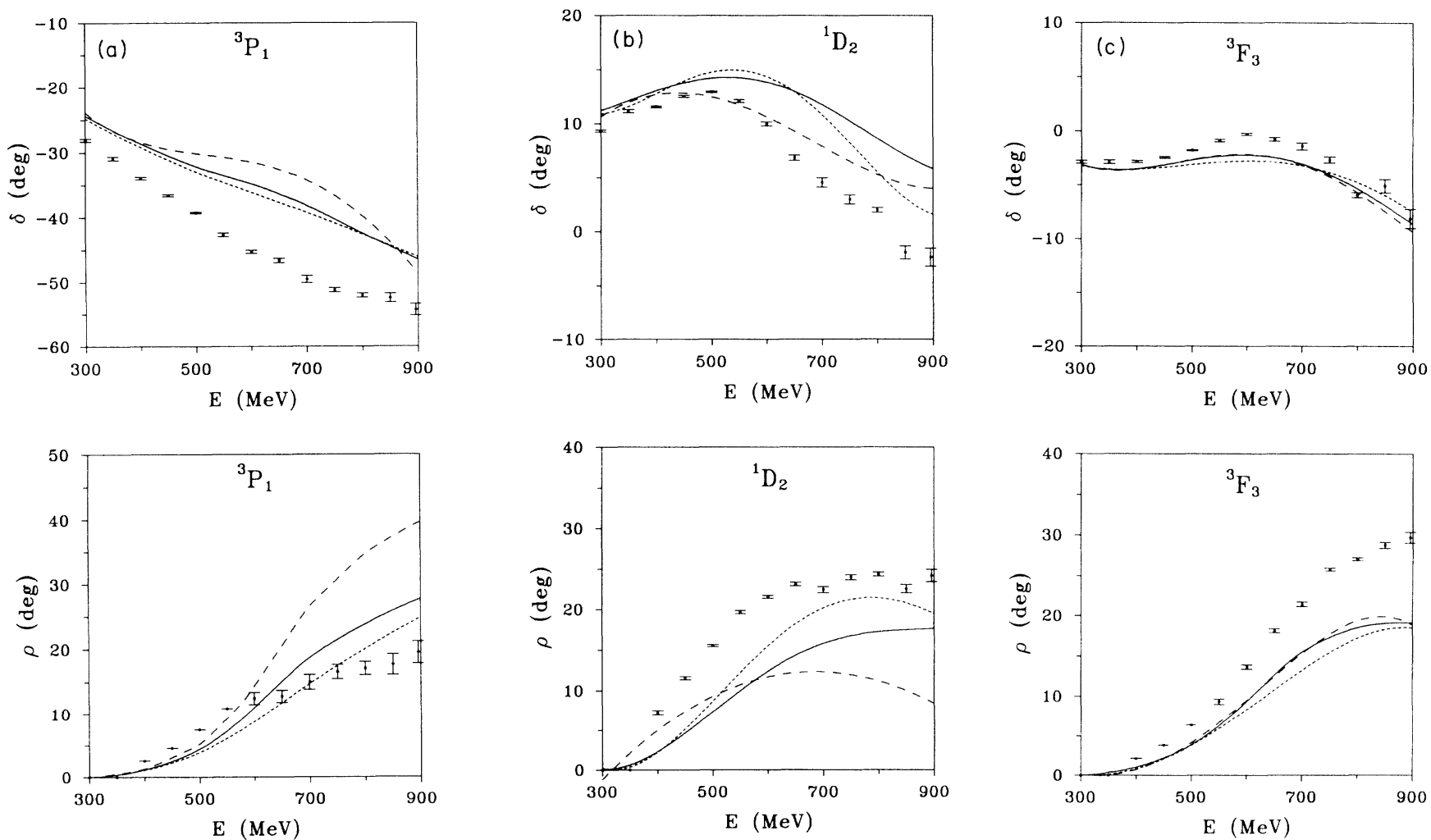

FIG. 13. Two-nucleon phase shifts $\delta$ and inelasticities $\rho$ as a function of the nucleon laboratory kinetic energy. The scattering parameters in the uncoupled partial waves ${ }^{3} P_{1},{ }^{1} D_{2}$, and ${ }^{3} F_{3}$ are shown. The curves have the same meaning as in Fig. 9. The experimental phase shifts are defined with respect to Coulomb-distorted plane waves; the calculation omits the Coulomb potential. This difference is immaterial at the considered energies. The experimental phase shifts are taken from Ref. [30]. 

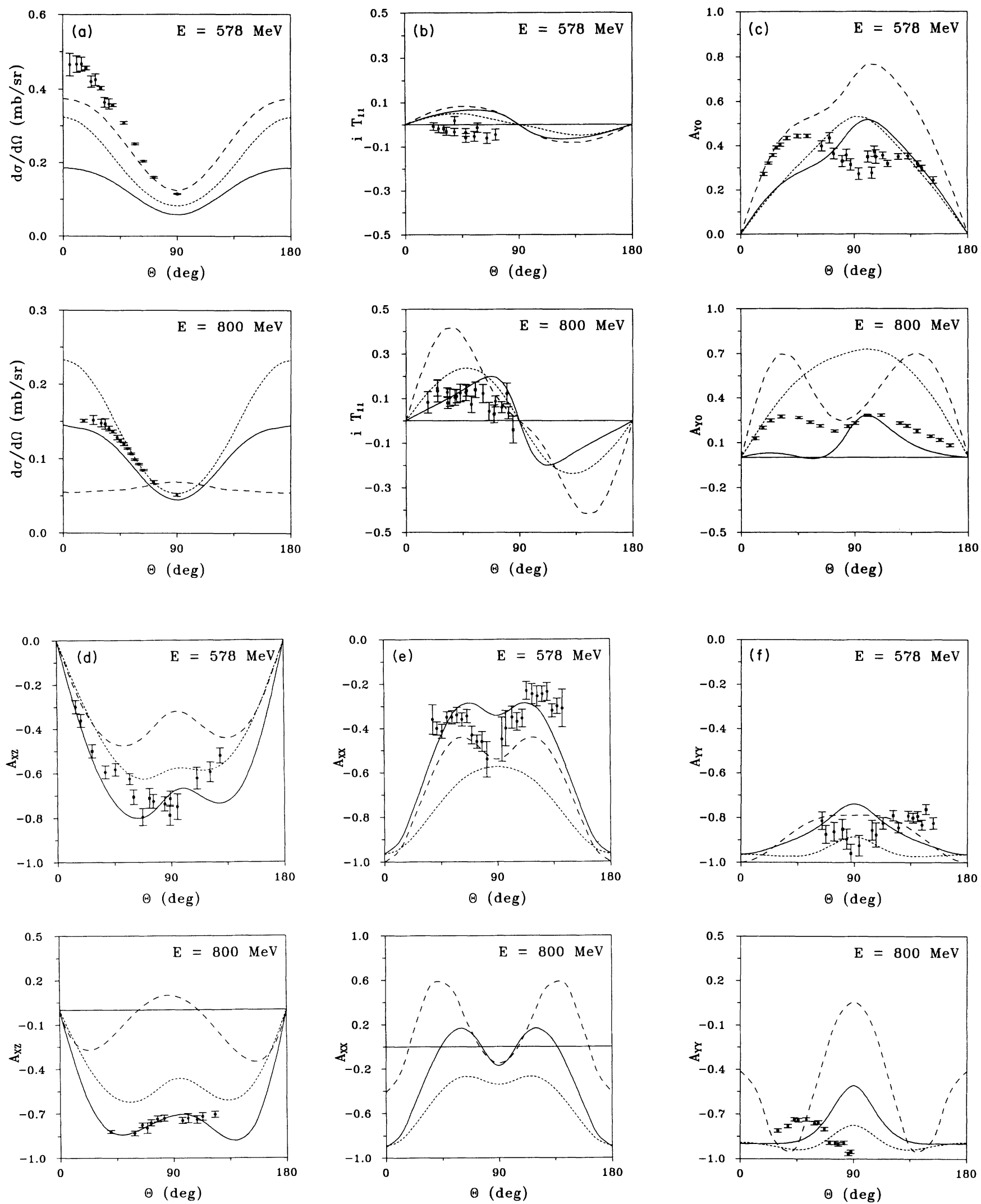

FIG. 14. Differential cross section $d \sigma / d \Omega$ and selected spin observables for the reaction $p p \rightarrow \pi^{+} d$ at the incident proton laboratory kinetic energies 578 and $800 \mathrm{MeV}$ as a function of the pion c.m. angle $\theta$. The curves have the same meaning as in Fig. 9. The data are taken from Refs. [31] and [32]. 

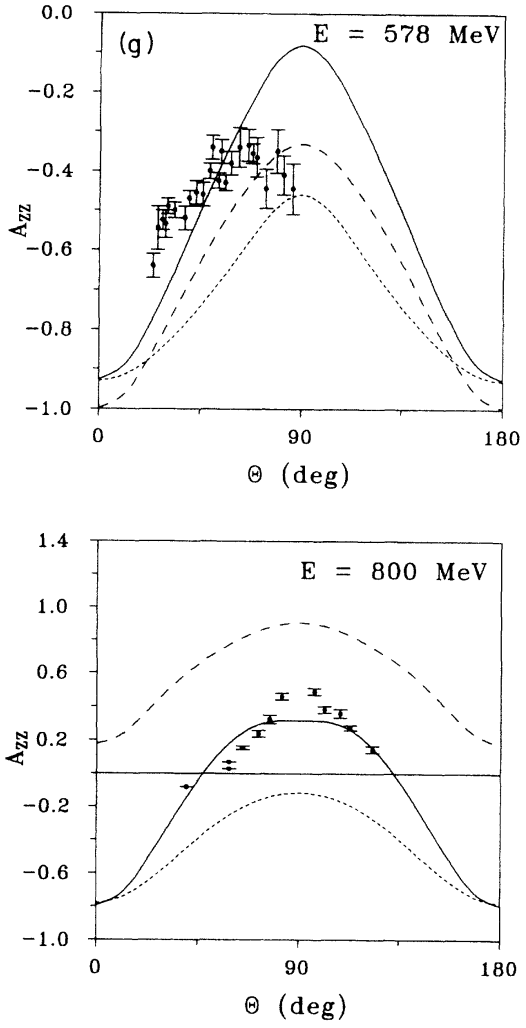

FIG. 14. (Continued).

the reference results are identical ${ }^{1}$ to the corresponding ones of Ref. [15].

\section{A. Total cross sections}

Total spin-averaged and spin-dependent cross sections are shown in Figs. 9 and 10 for two-nucleon scattering. The total proton-proton reaction cross section is far too small at high energies; the improvement by the nucleon$\Delta$ potentials is minor. In contrast, the spin-dependent proton-proton reaction cross sections get remarkably improved by the inclusion of the meson-exchange nucleon- $\Delta$ potential, which also does well for the reaction $p p \rightarrow \pi^{+} d$ according to Fig. 11. In contrast, the total pion-deuteron reaction cross sections are better described by the quarkmodel nucleon- $\Delta$ potential, as can be seen in Fig. 12.

\section{B. Elastic nucleon-nucleon scattering}

Figure 13 shows the effect of the two nucleon- $\Delta$ potentials on the nucleon-nucleon phase shifts and inelastici-

\footnotetext{
${ }^{1}$ Reference [15] used the real part of the energy-dependent pion-exchange interaction $P_{\Delta} H_{1}(z) P_{\Delta}$ only. All results of this paper were therefore obtained first with the approximation, and those results are plotted in this paper. However, all results were now also recalculated without that approximation, keeping $P_{\Delta} H_{1}(z) P_{\Delta}$ in full with its real and imaginary parts. The difference in results is almost invisible except for the differential cross section of elastic pion-deuteron scattering in Fig. 16(a); all results of Fig. 16 refer to that improved calculation, in contrast to the other figures in this paper.
}
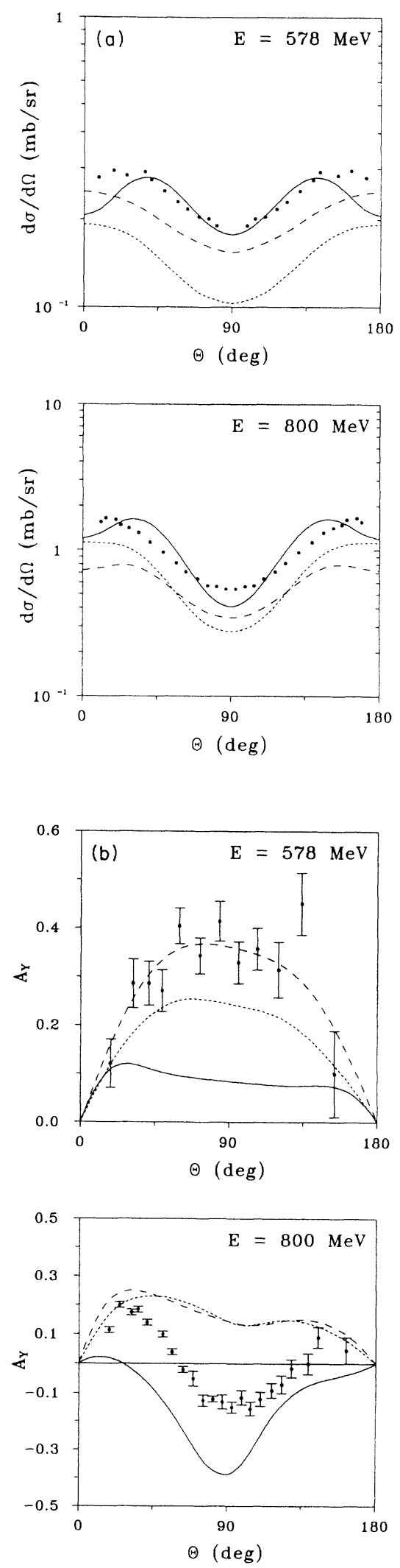

FIG. 15. Differential cross section $d \sigma / d \Omega$ and beam asymmetry $A_{y}$ for the reaction $p p \rightarrow n \Delta^{++}\left(p \pi^{+}\right)$at the incident proton laboratory kinetic energies 578 and $800 \mathrm{MeV}$ as a function of the $\Delta^{++}$c.m. scattering angle $\theta$. The curves have the same meaning as in Fig. 9 the data are taken from Ref. [6]. 

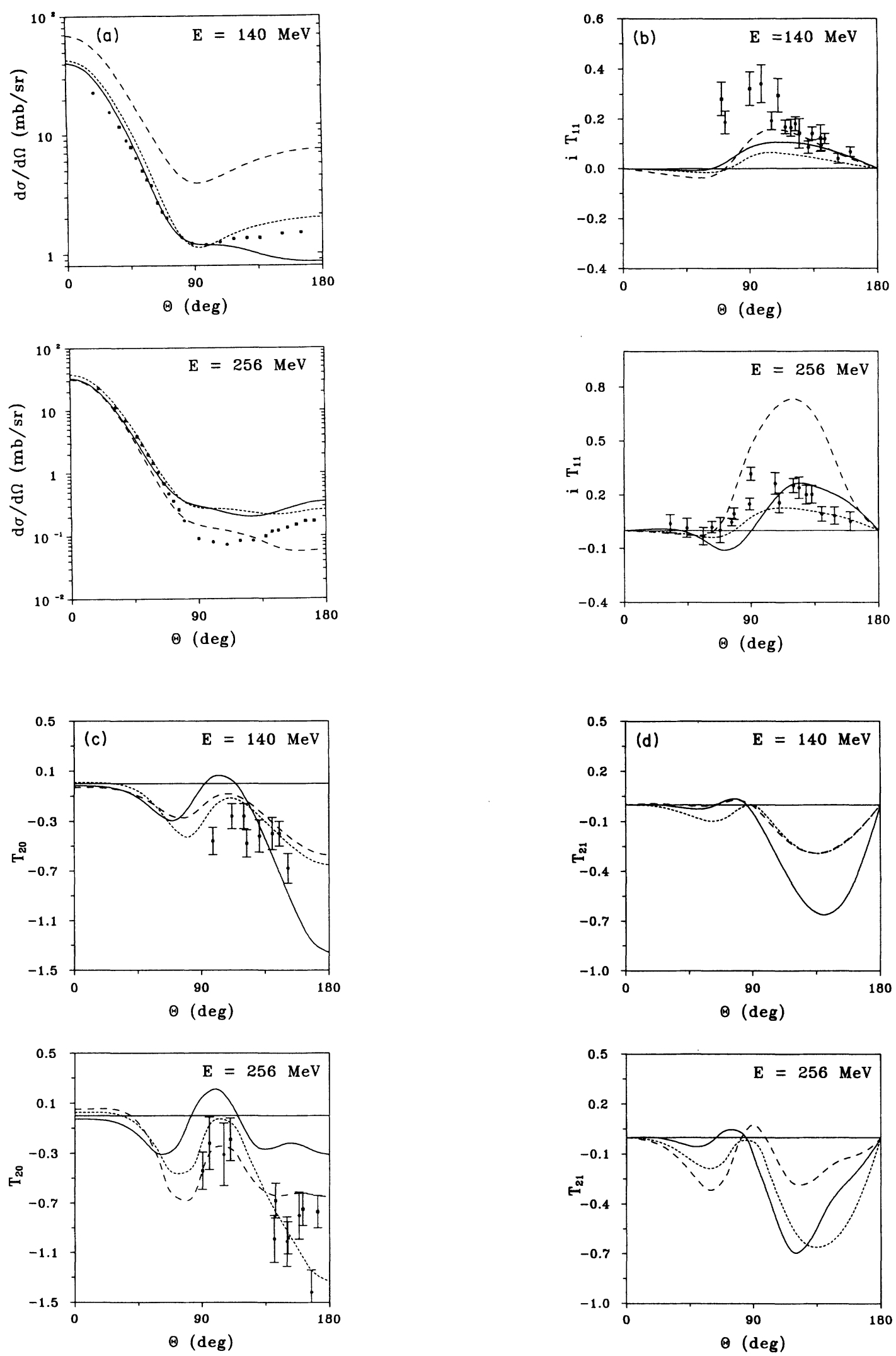

FIG. 16. Differential cross section $d \sigma / d \Omega$ are selected spin observable for elastic pion-deuteron scattering at the pion laboratory kinetic energies 140 and $256 \mathrm{MeV}$ as a function of the pion c.m. angle $\theta$. The curves have the same meaning as in Fig. 9. The data are taken from Refs. [34] and [35]. 
ties in three partial waves, i.e., ${ }^{3} P_{1},{ }^{1} D_{2}$, and ${ }^{3} F_{3}$. By construction the employed force models are almost phase equivalent with the purely nucleonic reference potential $V_{N N}$ of Eq. (3.1), i.e., the Paris potential in the calculations of this paper, as Fig. 7 proves. This is the reason why phase shifts are inelasticities below $300 \mathrm{MeV}$ nucleon laboratory kinetic energy are not displayed.

Phase shift parameters are extracted from the on-shell transition matrix elements in all important partial waves. The inclusion of a nucleon- $\Delta$ potential yields a solid effect in the ${ }^{3} P_{1}$ and ${ }^{1} D_{2}$ partial waves; the effect is larger and more structured for the meson-exchange nucleon- $\Delta$ potential; the inelasticity in ${ }^{1} D_{2}$ is decreased; the enhanced decrease for the meson-exchange nucleon- $\Delta$ potential may be due to its strong tensor coupling as Ref. [15] suggests. In all other partial waves considered, the effect is small; the ${ }^{3} F_{3}$ partial wave is shown as an example. Improvement of the theoretical predictions compared with experimental data is, however, never globally achieved. The smooth quark-model nucleon- $\Delta$ potential affects nucleon-nucleon scattering by very little; its strong short-range repulsion, i.e., in the isotriplet ${ }^{3} S_{1}$ nucleon- $\Delta$ partial wave, is not effective in nucleonnucleon scattering, since it does not couple.

\section{Pion production in nucleon-nucleon scattering}

Observables of the two reactions $p p \rightarrow \pi^{+} d$ and $p p \rightarrow$ $n \Delta^{++}\left(p \pi^{+}\right)$in proton-proton scattering are discussed. In the second process, three-body final states are only considered in the kinematic regime in which the proton and the positive pion form a $P_{33}$ resonance $\Delta^{++}[6]$. Theoretical predictions for the spin-averaged and spin-dependent differential cross sections are calculated for the two proton laboratory kinetic energies 578 and $800 \mathrm{MeV}$; they are displayed for the first reaction in Fig. 14 and for the second in Fig. 15.

Most observables of both reactions show strong sensitivity with respect to the inclusion of a nucleon- $\Delta$ potential as already noted in Ref. [33]. Exceptions are the tensor polarizations $T_{2 m}$ in $p p \rightarrow \pi^{+} d$, which remain pretty unaffected and which are therefore not shown. The changes with energy are milder for the quark-model potential which has less failures in its predictions of data, especially at higher energies, though it cannot claim broad success either. In contrast, the predictions based on the meson-exchange nucleon- $\Delta$ potential get even worse at higher energies.

\section{Elastic pion-deuteron scattering}

Observables of elastic pion-deuteron scattering are calculated for the pion laboratory kinetic energies 140 and $265 \mathrm{MeV}$. They are displayed in Fig. 16. In piondeuteron scattering, the isotriplet nucleon- $\Delta{ }^{3} S_{1}$ partial wave can operate. Its strong short-range repulsion in the quark-model potential may be responsible for the fact that the differential cross section is not deepened with increasing energy. The effect is in contrast to the spectacular improvements seen by Ferreira and Dosch [19] for a similar interaction, however, seen there in the qualita- tively insufficient Born approximation treatment of the nucleon- $\Delta$ potential. Again, the effects of the quarkmodel nucleon- $\Delta$ potential are milder and less energy dependent compared with those of the meson-exchange nucleon- $\Delta$ potential.

\section{E. Pion-deuteron breakup}

Pion-induced breakup of the deuteron is considered in the kinematic regime in which the proton and positive pion form a $P_{33}$ resonance $\Delta^{++}$, i.e., in the reaction $\pi^{+} d \rightarrow n \Delta^{++}\left(p \pi^{+}\right)$. Only the differential cross section is calculated for the pion laboratory kinetic energies 140 and $256 \mathrm{MeV}$. The results are documented in Fig. 17. Experimental data do not exist yet. The differential cross section does not indicate any pronounced dependence on the nucleon- $\Delta$ interaction as already observed in Ref. [36].

The results of Secs. IV A-IV E indicate a strong sensitivity of many observables in the two-nucleon system above the pion threshold on the inclusion of an instantaneous nucleon- $\Delta$ potential. Two models of the nucleon$\Delta$ potential were studies and their predictions compared, one based on quark-model dynamics, one on meson exchange. Compared with theoretical predictions without its inclusion, there are some improvements by either of the two choices and some dramatic failures by both. Thus neither model for the nucleon- $\Delta$ potential can be pre-
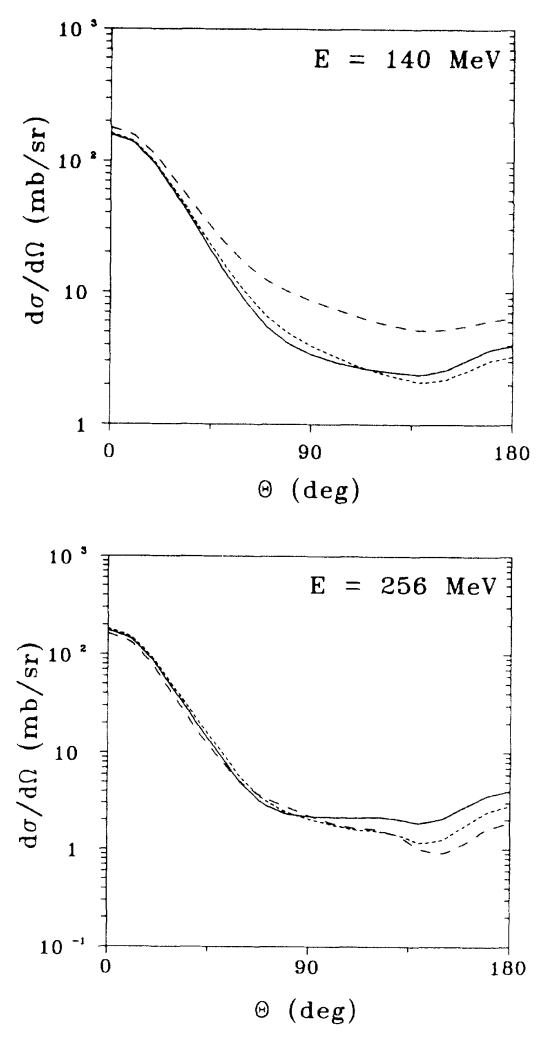

FIG. 17. Differential cross section $d \sigma / d \Omega$ for the breakup reaction $\pi^{+} d \rightarrow n \Delta^{++}\left(p \pi^{+}\right)$at the pion laboratory kinetic energies 140 and $256 \mathrm{MeV}$ as a function of the $\Delta^{++}$c.m. angle $\theta$. The curves have the same meaning as in Fig. 9. 
ferred over the other. One has also to keep in mind that any spin-orbit contribution is still left out. The sensitivity of observables on the inclusion of a nucleon- $\Delta$ potential may be felt to be disturbing; we consider it encouraging: The sensitivity will eventually allow the nucleon- $\Delta$ potential to be tuned to the data. Such a program for tuning the nucleon- $\Delta$ potential remains at present out of reach, since calculations are still too complicated and not streamlined yet to base a fit on them. Nevertheless, such a project should be aimed at the future.

\section{ACKNOWLEDGMENTS}

The authors acknowledge enlightening discussions with A. Buchmann and U. Straub on the employed quark model and K. Bugaev's help in the recalculation described in the footnote of Sec. IV. A.V. thanks P. González for critical advice; he thanks the Nuclear Theory Group of the University of Hannover for the kind hospitality extended to him during several stays during which this work was done. The work was funded by Dirección General de Investigación Científica y Técnica (DGICYT) under Contract No. PB91-0119-C02-02, by the Deutsche Forschungs Gemeinschaft (DFG) under Contract No. Sa247/7-2, by the German Federal Minister for Research and Technology (BMFT) under Contract No. $06 \mathrm{OH}$ 752, and by the German Academic Exchange Service (DAAD) under Contract No. 303-INIDA-dr. The calculations for this paper were performed at "Regionales Rechenzentrum für Niedersachsen."
[1] A. M. Green, Rep. Prog. Phys. 39, 1109 (1976).

[2] P. U. Sauer, Prog. Nucl. Part. Phys. 16, 35 (1986).

[3] M. Hirata, F. Lenz, and K. Yazaki, Ann. Phys. (N.Y.) 108, 116 (1977).

[4] T.-S. H. Lee and K. Ohta, Phys. Rev. C 25, 3043 (1982).

[5] R. L. Shypit et al., Phys. Rev. Lett. 60, 901 (1988).

[6] A. B. Wicklund et al., Phys. Rev. D 35, 2670 (1987).

[7] F. Fernández, A. Valcarce, U. Straub, and A. Faessler, J. Phys. G 19, 2013 (1993).

[8] F. Fernández and E. Oset, Nucl. Phys. A455, 720 (1986).

[9] K. Bräuer, A. Faessler, F. Fernández, and K. Shimizu, Nucl. Phys. A507, 599 (1990).

[10] K. Shimizu, Rep. Prog. Phys. 52, 1 (1989).

[11] A. Buchmann, E. Hernández, and K. Yazaki, Phys. Lett. B 269, 35 (1991).

[12] M. Oka and K. Yazaki, in Quarks and Nuclei, International Review of Nuclear Physics, edited by W. Weise (World Scientific, Singapore, 1984), Vol. 1.

[13] K. Holinde, Nucl. Phys. A415, 477 (1984).

[14] M. T. Peña, H. Henning, and P. U. Sauer, Phys. Rev. C 42, 855 (1990).

[15] M. T. Peña, H. Garcilazo, U. Oelfke, and P. U. Sauer, Phys. Rev. C 45, 1487 (1992).

[16] G. E. Brown and W. Weise, Phys. Rep. C 22, 279 (1975).

[17] M. Harvey, Nucl. Phys. A352, 301 (1981).

[18] S. Saito, Prog. Theor. Phys. 41, 705 (1969).

[19] E. Ferreira and H. G. Dosch, Phys. Rev. C 40, 1750 (1989).

[20] T. Takaki and M. Thies, Phys. Rev. C 38, 2230 (1988).

[21] E. O. Alt, P. Grassberger, and W. Sandhas, Nucl. Phys. B2, 167 (1967).

[22] H. Pöpping, P. U. Sauer, and Zhang Xi-Zhen, Nucl. Phys.
A474, 557 (1987); A550, 563(E) (1992).

[23] A. Bulla and P. U. Sauer, Few-Body Sys. 12, 141 (1992).

[24] M. Lacombe, B. Loiseau, J. M. Richard, R. Vinh Mau, J. Côé, P. Pirés, and R. de Tourreil, Phys. Rev. C 21, 861 (1980).

[25] F. Shimizu, Y. Kubota, H. Koiso, F. Sai, S. Sakamoto, and S. S. Yamamoto, Nucl. Phys. A386, 571 (1982); V. Flamino et al., Report No CERN-HERA 84-01, 1984.

[26] E. Aprile-Giboni et al., Phys. Rev. D 28, 21 (1983); E. Aprile-Giboni et al., Nucl. Phys. A431, 637 (1984); I. P. Auer et al., Phys. Rev. Lett. 51, 1141 (1983); 51, 1814(E) (1983); see also references to some data in M. P. Locher et al., Adv. Nucl. Phys. 17, 47 (1986).

[27] J. A. Niskanen, Phys. Lett. 141B, 301 (1984).

[28] C. Richard-Serre, W. Hirt, D. F. Measday, E. G. Michaelis, M. J. Saltmarsh, and P. Skarek, Nucl. Phys. B20, 413 (1970).

[29] E. Pedroni et al., Nucl. Phys. A300, 321 (1978).

[30] R. A. Arndt, L. D. Roper, R. L. Workman, and M. W. McNaughton, Phys. Rev. D 45, 3995 (1992).

[31] A. B. Laptev and I. I. Strakovsky, Leningrad Nuclear Physics Institute Report, 1985.

[32] G. R. Smith et al., Phys. Rev. C 30, 980 (1984).

[33] C. Alexandrou and B. Blankleider, Phys. Rev. C 42, 517 (1990).

[34] R. Gabathuler et al., Nucl. Phys. A350, 253 (1980); C. Ottermann et al., Phys. Rev. C 32, 928 (1985).

[35] G. R. Smith et al., Phys. Rev. C 29, 2206 (1984); E. Ungricht et al., ibid. 31, 934 (1985); Y. M. Shin et al., Phys. Rev. Lett. 55, 2672 (1985).

[36] H. Garcilazo, Phys. Rev. C 42, 2334 (1990). 